\title{
SUMMARY STATISTICS FOR ENDPOINT-CONDITIONED CONTINUOUS-TIME MARKOV CHAINS
}

\author{
ASGER HOBOLTH * AND \\ JENS LEDET JENSEN,** Aarhus University
}

\begin{abstract}
Continuous-time Markov chains are a widely used modelling tool. Applications include DNA sequence evolution, ion channel gating behaviour, and mathematical finance. We consider the problem of calculating properties of summary statistics (e.g. mean time spent in a state, mean number of jumps between two states, and the distribution of the total number of jumps) for discretely observed continuous-time Markov chains. Three alternative methods for calculating properties of summary statistics are described and the pros and cons of the methods are discussed. The methods are based on (i) an eigenvalue decomposition of the rate matrix, (ii) the uniformization method, and (iii) integrals of matrix exponentials. In particular, we develop a framework that allows for analyses of rather general summary statistics using the uniformization method.
\end{abstract}

Keywords: Continuous-time Markov chain; dwelling time; EM algorithm; transition number; uniformization

2010 Mathematics Subject Classification: Primary 60-08

Secondary 60J22; 60J25; 60J27

\section{Motivation and background}

We review and extend three ways of calculating conditional properties of summary statistics of a continuous-time Markov chain (CTMC). The conditioning is with respect to the starting point $x(0)$ and the ending point $x(T)$ of a process $x(t)$ considered in the interval $0 \leq t \leq T$. Typical summary statistics are either the time $D_{\alpha}$ spent in a state $\alpha$ or the number of transitions $N_{\alpha \beta}$ from state $\alpha$ to state $\beta$. Since, generally, for a statistic $H$, we have

$$
\mathrm{E}[H \mid x(0)=a, x(T)=b]=\frac{\mathrm{E}[H 1(x(T)=b) \mid x(0)=a]}{\mathrm{P}(x(T)=b \mid x(0)=a)},
$$

we formulate our results through terms of the form $\mathrm{E}[H 1(x(T)=b) \mid x(0)=a]$. The first approach to calculating the conditional properties is through an eigenvalue decomposition of the rate matrix of the process, and will only be mentioned briefly (Section 2). The second approach (Section 3), based on the uniformization method, will be treated in more detail, and in particular we derive new formulae for various covariance terms. The uniformization method is the most general of the three methods as it allows for calculation of moments and distributions of rather general summary statistics. The third approach (Section 4) seems to be less known and is based on Van Loan's (1978) method of calculating integrals involving the matrix exponential.

Received 27 May 2010; revision received 28 March 2011.

* Postal address: Bioinformatics Research Center, Aarhus University, C. F. Møllers Alle 8, DK-8000 Aarhus C, Denmark. Email address: asger@birc.au.dk

** Postal address: Department of Mathematical Sciences, Aarhus University, Ny Munkegade Bldg 1530, DK-8000 Aarhus C, Denmark. Email address: jlj@imf.au.dk 
In this introductory section we describe a few applications where conditional properties of summary statistics are needed. First, we review the very important problem of estimating the transition rates of a CTMC from observations at a finite set of time points using the expectationmaximization (EM) algorithm (Subsection 1.1). We identify five endpoint-conditioned mean values needed in the EM algorithm. Second, we consider summary statistics arising from the use of CTMCs in molecular biology (Subsection 1.2).

\subsection{Summary statistics needed in the EM algorithm}

We consider a CTMC $x(t)$ with states $\{1,2, \ldots, m\}$ defined by a rate matrix $Q=\left(q_{i j}\right)$, where $q_{i i}=-\sum_{j \neq i} q_{i j}$. When the process is observed at discrete time points $0=s_{0}<$ $s_{1}<s_{2}<\cdots<s_{k}=\tau$ only, maximization of the likelihood function is often done via the EM algorithm. Let $y=\left(x\left(s_{0}\right), x\left(s_{1}\right), \ldots, x\left(s_{k}\right)\right)$ be the actual observations. As we describe below, the expectation step of the algorithm gives rise to endpoint-conditioned mean values. Furthermore, for finding the asymptotic variance of the maximum likelihood estimates, we need endpoint-conditioned second-order moments.

The full log-likelihood function $\ell(Q)$ based on continuous observations in the interval $0 \leq$ $t \leq \tau$ is given by (see, e.g. Guttorp (1995, Chapter 3.7))

$$
\ell(Q ;\{x(t): 0 \leq t \leq \tau\})=-\sum_{\alpha=1}^{m} D_{\alpha}(0, \tau) q_{\alpha \alpha}+\sum_{\alpha \neq \beta} N_{\alpha \beta}(0, \tau) \log q_{\alpha \beta},
$$

where $D_{\alpha}(0, \tau)$ is the time spent in state $\alpha$ and $N_{\alpha \beta}(0, \tau)$ is the number of jumps from state $\alpha$ to state $\beta$. The EM algorithm (see Dempster et al. (1977)) is an iterative procedure consisting of an expectation step (E-step) and a maximization step (M-step). In the E-step of the algorithm we calculate $G\left(Q ; Q_{0}\right)=\mathrm{E}_{Q_{0}}[\ell(Q) \mid y]$, and in the M-step a new parameter value $Q_{1}$ is obtained as the value that maximizes $G\left(Q ; Q_{0}\right)$. The algorithm converges to a local maximum of the data likelihood $\ell(Q ; y)$. The simple structure of the full log likelihood (1) implies that the M-step is easy.

Let $A(0, \tau)$ be either $D_{\alpha}(0, \tau)$ or $N_{\alpha \beta}(0, \tau)$. In the E-step we must calculate $\mathrm{E}[A(0, \tau) \mid y]$. Since $A$ is an additive statistic, $A(0, \tau)=\sum_{i=1}^{k} A\left(s_{i-1}, s_{i}\right)$, and because of the Markov property, we have

$$
\mathrm{E}[A(0, \tau) \mid y]=\sum_{i=1}^{k} \mathrm{E}\left[A\left(s_{i-1}, s_{i}\right) \mid x\left(s_{i-1}\right), x\left(s_{i}\right)\right] .
$$

Thus, the EM algorithm requires the calculation of endpoint-conditioned mean values.

For calculating the asymptotic variance of the maximum likelihood estimates, we also need second-order moments of the form $\mathrm{E}[A(0, \tau) \tilde{A}(0, \tau) \mid y]$, where again $A(0, \tau)$ and $\tilde{A}(0, \tau)$ are additive statistics of the form $D_{\alpha}(0, \tau)$ or $N_{\alpha \beta}(0, \tau)$ (see, e.g. Hobolth and Jensen (2005)). Once more, because of the additive structure and the Markov property, we end up with endpointconditioned, second-order moments of the form $\mathrm{E}\left[A\left(s_{i-1}, s_{i}\right) \tilde{A}\left(s_{i-1}, s_{i}\right) \mid x\left(s_{i-1}\right), x\left(s_{i}\right)\right]$.

Summarizing, inference in a CTMC via the EM algorithm requires the calculation of the following endpoint-conditioned mean values:

$$
\begin{gathered}
\mathrm{E}\left[D_{\alpha} 1(x(T)=b) \mid x(0)=a\right], \\
\mathrm{E}\left[N_{\alpha \beta} 1(x(T)=b) \mid x(0)=a\right], \\
\mathrm{E}\left[D_{\alpha} D_{\beta} 1(x(T)=b) \mid x(0)=a\right],
\end{gathered}
$$




$$
\begin{gathered}
\mathrm{E}\left[N_{\alpha \beta} N_{\gamma \delta} 1(x(T)=b) \mid x(0)=a\right], \\
\mathrm{E}\left[N_{\alpha \beta} D_{\gamma} 1(x(T)=b) \mid x(0)=a\right],
\end{gathered}
$$

where $D_{\alpha}=D_{\alpha}(0, T)$ and $N_{\alpha \beta}=N_{\alpha \beta}(0, T)$. All the abovementioned mean values have integral representations which are linear combinations of the following terms (see, e.g. Hobolth and Jensen (2005)):

$$
\begin{gathered}
I(a, b, \alpha, \beta)=\int_{0}^{T} P_{a \alpha}(t) P_{\beta b}(T-t) \mathrm{d} t, \\
I(a, b, \alpha, \beta, \gamma, \delta)=\int_{0}^{T} \int_{0}^{t} P_{a \alpha}(u) P_{\beta \gamma}(t-u) P_{\delta b}(T-t) \mathrm{d} u \mathrm{~d} t,
\end{gathered}
$$

where $P_{\alpha \beta}(t)=(\exp (Q t))_{\alpha \beta}$ is the transition probability. In particular, we note that the mean value (2a) of the time spent in state $\alpha$ is given by $I(a, b, \alpha, \alpha)$ and the mean value (2b) of the number of jumps from state $\alpha$ to state $\beta$ is given by $q_{\alpha \beta} I(a, b, \alpha, \beta)$.

Further discussion of transition rate estimation based on incomplete observations can be found in Metzner et al. (2007) and the references therein. Bladt and Sørensen (2009) described an application in mathematical finance. They considered the problem of estimating transition rates between credit ratings from observations at discrete points (e.g. weekly observations). Holmes and Rubin (2002), Hobolth and Jensen (2005), and Kosiol et al. (2007) used the EM algorithm for analysis of DNA sequence data. The DNA sequences were observed at present day and related by a phylogenetic tree. CTMCs are not exclusively an important modelling tool in mathematical finance and molecular evolution; for example, Ball and Milne (2005) described how insights into the gating mechanism of a single ion channel can be obtained by modelling the system using a CTMC on a finite state space.

\subsection{Further summary statistics used in applications of CTMCs}

Let $R$ denote a set of transitions of interest, and let $N_{R}=\sum_{(\alpha, \beta) \in R} N_{\alpha \beta}$ be the number of such transitions. Minin and Suchard (2008) described how the distribution of $N_{R}$ is of interest in evolutionary developmental biology. They considered in particular a CTMC along a small evolutionary tree with observations at the tips of the tree. For the case of a two-state Markov chain, they derived closed-form expressions for $\left.\mathrm{P}\left(N_{R}=k, x(T)=b\right) \mid x(0)=a\right)$. For the general case, they considered moments of $N_{R}$. As an example, they considered the mean number of transitions and transversions within a small multiple alignment of DNA sequences. Note that the moments of $N_{R}$ can be found through the moments of $N_{\alpha \beta},(\alpha, \beta) \in R$. As a step in their analysis, Minin and Suchard (2008) expressed the moment generating function of $N_{R}$ as a matrix exponential. More generally, the joint Laplace transform and moment generating function of all the $D_{\alpha} \mathrm{s}$ and $N_{\alpha \beta}$ s are given as a matrix exponential in Bladt et al. (2002).

In an evolutionary setting Siepel et al. (2006) also considered the distribution $\mathrm{P}\left(N_{R}=k\right.$, $x(T)=b) \mid x(0)=a$ ) for the case where $R$ is the set of all transitions. These authors used the statistic $N_{R}$ to identify lineages in a phylogenetic tree that are under selection in a specific genomic region.

\section{The eigenvalue decomposition method}

The transition matrix $P(t)$ is given by the matrix exponential $P(t)=\exp (Q t)$. If $Q$ is diagonalizable with real eigenvalues $\lambda_{1}, \ldots, \lambda_{m}$, we have $Q=U \Lambda U^{-1}$, where $\Lambda=$ $\operatorname{diag}\left(\lambda_{1}, \ldots, \lambda_{m}\right)$ is a diagonal matrix with the eigenvalues on the diagonal. In this case the integrals in (3) and (4) can be evaluated easily. In particular, $P(t)=U \exp (\Lambda t) U^{-1}$ and we 
obtain

$$
\begin{aligned}
I(a, b, \alpha, \beta) & =\int_{0}^{T} P_{a \alpha}(t) P_{\beta b}(T-t) \mathrm{d} t \\
& =\int_{0}^{T}\left(U \exp (\Lambda t) U^{-1}\right)_{a \alpha}\left(U \exp (\Lambda(T-t)) U^{-1}\right)_{\beta b} \mathrm{~d} t \\
& =\sum_{i} U_{a i} U_{i \alpha}^{-1} \sum_{j} U_{\beta j} U_{j b}^{-1} \int_{0}^{T} \mathrm{e}^{t \lambda_{i}+(T-t) \lambda_{j}} \mathrm{~d} t .
\end{aligned}
$$

The last integral is easy to evaluate in the case of real eigenvalues.

These formulae were given in Holmes and Rubin (2002) for (3) and Hobolth and Jensen (2005) for (4), and correspond to the formulae in Minin and Suchard (2008) for the statistic $N_{R}$ using the fact that $N_{R}=\sum_{(\alpha, \beta) \in R} N_{\alpha \beta}$. A reversible process always admits an eigenvalue decomposition with real eigenvalues.

Hobolth and Jensen (2005) also considered the case where some of the eigenvalues are complex numbers and illustrated the results for the case of four states, corresponding to the four possible nucleotides $\{A, G, C, T\}$. Klosterman et al. (2006) in their supplementary material gave a general description of the eigenvalue decomposition method in the case of complex eigenvalues. When the rate matrix $Q$ is no longer diagonalizable, it is possible to use a Jordan decomposition. However, in this case the calculations for evaluating the integrals in (3) and (4) become more involved.

\section{The uniformization method}

The uniformization method was originally introduced as a way of calculating $P(t)=$ $\exp (Q t)$ (see Jensen (1953)). Let $\mu=\max _{i}\left(-q_{i i}\right)$, and define the discrete-time Markov chain (DTMC) transition matrix $R=Q / \mu+I$. Then

$$
\exp (Q t)=\exp (\mu(R-I) t)=\sum_{n=0}^{\infty} R^{n} \frac{(\mu t)^{n}}{n !} \mathrm{e}^{-\mu t}=\sum_{n=0}^{\infty} R^{n} \operatorname{Pois}(n ; \mu t)
$$

where Pois $(n ; \lambda)$ is the probability from a Poisson distribution with mean $\lambda$. More fundamentally, the uniformization method gives rise to an alternative description of the process itself. Let $z_{0}, z_{1}, \ldots$ be a Markov chain with transition matrix $R$. Independent of the chain, let $0=T_{0}<T_{1}<T_{2}<\cdots$ be the times of a Poisson process with rate $\mu$. Next, we define $\{x(t), t \geq 0\}$, by setting $x(t)=z_{k}$ in the time interval $T_{k} \leq t<T_{k+1}$. It is clear that the construction is equivalent to a CTMC with rates $\mu R_{i j}=q_{i j}, j \neq i$. For more details on the equivalence between a CTMC and a DTMC subordinated to a Poisson process, we refer the reader to Ross (1983, Section 5.8).

Consider now a statistic $H$ defined on $\{x(t), 0 \leq t \leq T\}$, which can be written as a function of the number of Poisson events $J$, the times $0=T_{0}<T_{1}<\cdots<T_{J}<T$, and the states $z_{0}, z_{1}, \ldots, z_{J}$. To study the properties of $H$, we condition on the value of $J$, and use the facts that the times are the ordered values of uniformly distributed times and that the states are from a Markov chain with transition matrix $R$. Also, conditioned on $J=n$, we generally use a recursion in $n$ to evaluate the properties of $H$. As an illustration, consider the original use of 
the uniformization method

$$
\begin{aligned}
\mathrm{P}(x(T)=b \mid x(0)=a) & =\sum_{n=0}^{\infty} \mathrm{P}\left(z_{n}=b \mid z_{0}=a\right) \operatorname{Pois}(n ; \mu T) \\
& =\sum_{n=0}^{\infty}\left(R^{n}\right)_{a b} \operatorname{Pois}(n ; \mu T),
\end{aligned}
$$

where $R^{n}$ can be calculated by the recursion $R^{n+1}=\left(R^{n}\right) R$.

What kind of general statistic $H$ do we want to consider? The class of statistics must contain the time spent in a state $D_{\alpha}=\sum_{i=0}^{J} 1\left(z_{i}=\alpha\right)\left(T_{i+1}-T_{i}\right)$, where $T_{J+1}=T$, as well as the number of transitions between two states $N_{\alpha \beta}=\sum_{i=1}^{J} 1\left(\left(z_{i-1}, z_{i}\right)=(\alpha, \beta)\right)$. These two cases are covered by a general statistic of the form

$$
H=\psi\left(z_{0}\right) f\left(T_{1}\right)+\sum_{i=1}^{J} \phi\left(z_{i-1}, z_{i}\right) f\left(T_{i+1}-T_{i}\right),
$$

where $f(t)=t$ and $\phi\left(z_{1}, z_{2}\right)=\psi\left(z_{2}\right)=1\left(z_{2}=\alpha\right)$ for $D_{\alpha}$, and $f \equiv 1, \psi(z) \equiv 0$, and $\phi\left(z_{1}, z_{2}\right)=1\left(\left(z_{1}, z_{2}\right)=(\alpha, \beta)\right)$ for $N_{\alpha \beta}$. Note also that the general form of $H$ in (6) need not be an additive statistic.

\subsection{Computing mean values using uniformization}

We want to find the mean of a general statistic $\mathrm{E}[H 1(x(T)=b) \mid x(0)=a]$ with $H$ defined in (6). We are particularly interested in two special cases: (i) where $H=D_{\alpha}$ and (ii) where $H=N_{\alpha \beta}$. In Theorem 1 below we treat the general case, and in Corollaries 1 and 2 below we consider the two special cases. Theorem 1 shows how the uniformization method allows a division of the conditional mean problem into three components that are each easy to handle. The components are concerned with properties of the number of jumps $n$, the interarrival times $T_{i+1}-T_{i}$, and the discrete Markov chain $\left(z_{0}, \ldots, z_{n}\right)$.

First we introduce some notation. Let $M(n)$ be the $m \times m$ matrix with entries

$$
M(n, a, b)=\mathrm{E}\left[1\left(z_{n}=b\right)\left(\psi\left(z_{0}\right)+\sum_{i=1}^{n} \phi\left(z_{i-1}, z_{i}\right)\right) \mid z_{0}=a\right],
$$

and let $\phi=(\phi(a, b))$. Also, let $\operatorname{diag}(\psi)$ be a diagonal matrix with entries $\psi(j), j=1, \ldots, m$. For two matrices $A$ and $B$, we let $A * B$ be the matrix with entries given by the product of the corresponding entries of $A$ and $B$. Finally, we define $\delta(n, T, f)=\mathrm{E}\left[f\left(T W_{n}\right)\right]$, where $W_{n}$ is a beta-distributed random variable with parameters $(1, n)$.

Theorem 1. (General statistic.) Let $H$ be the general statistic defined in (6). Then we have

$$
\mathrm{E}[H 1(x(T)=b) \mid x(0)=a]=\sum_{n=0}^{\infty} \delta(n, T, f) M(n, a, b) \operatorname{Pois}(n ; \mu T) .
$$

The matrix $M(n)$ is determined by the recursion

$$
M(n)=M(n-1) R+R^{n-1}(\phi * R), \quad n \geq 1,
$$

with starting value $M(0)=\operatorname{diag}(\psi)$. The solution of the recursion is

$$
M(n)=\operatorname{diag}(\psi) R^{n}+\sum_{\ell=0}^{n-1} R^{\ell}(\phi * R) R^{n-1-\ell} .
$$


Proof. The properties of the interarrival times $W_{i}=T_{i}-T_{i-1}$, conditioned on $J=n$ and with $T_{0}=0$ and $T_{n+1}=T$, can be studied as follows. Let $W_{1}, \ldots, W_{n+1}$ be independent, exponentially distributed variables with mean $1 / \mu$, and let $S_{n+1}=\sum_{i=1}^{n+1} W_{i}$ be the sum of these variables. Then the conditional distribution of the vector $T_{1}, T_{2}-T_{1}, \ldots, T_{n+1}-T_{n}$ given $J=n$ is the same as the conditional distribution of $W_{1}, W_{2}, \ldots, W_{n+1}$ given that $S_{n+1}=T$. In particular, we note that $\left(W_{1}, \ldots, W_{n+1}\right) / T$ conditional on $S_{n+1}=T$ follows a Dirichlet distribution with parameter $(1, \ldots, 1)$. Consequently, the marginal distribution of $W_{i} / T$ conditional on $S_{n+1}=T$ is a beta distribution with parameter $(1, n)$.

We now use the uniformization method:

$$
\begin{aligned}
& \mathrm{E}[H \\
& \quad=\mathrm{E}\left[\left(\psi\left(\psi\left(z_{0}\right) f\left(W_{1}\right)+\sum_{i=1}^{n} \phi\left(z_{i-1}, z_{i}\right) f\left(W_{i+1}\right)\right) 1\left(z_{n}=b\right) \mid z_{0}=a, S_{n+1}=T\right]\right. \\
& \quad=\delta(n, T, f) \mathrm{E}\left[1\left(z_{n}=b\right)\left(\psi\left(z_{0}\right)+\sum_{i=1}^{n} \phi\left(z_{i-1}, z_{i}\right)\right) \mid z_{0}=a\right] \\
& \quad=\delta(n, T, f) M(n, a, b) .
\end{aligned}
$$

Here

$$
M(0, a, b)=\mathrm{E}\left[\psi\left(z_{0}\right) 1\left(z_{0}=b\right) \mid z_{0}=a\right]=\psi(a) 1(a=b),
$$

and, for $n \geq 1$, we obtain the following recursion upon dividing the mean value according to the value of $z_{n-1}$ :

$$
\begin{aligned}
M(n, a, b)= & \mathrm{E}\left[1\left(z_{n}=b\right)\left(\psi\left(z_{0}\right)+\sum_{i=1}^{n} \phi\left(z_{i-1}, z_{i}\right)\right) \mid z_{0}=a\right] \\
= & \sum_{c=1}^{m} \mathrm{E}\left[1\left(z_{n}=b\right) 1\left(z_{n-1}=c\right)\right. \\
& \left.\times\left(\psi\left(z_{0}\right)+\sum_{i=1}^{n-1} \phi\left(z_{i-1}, z_{i}\right)+\phi\left(z_{n-1}, z_{n}\right)\right) \mid z_{0}=a\right] \\
= & \sum_{c=1}^{m} M(n-1, a, c) R_{c b}+\sum_{c=1}^{m}\left(R^{n-1}\right)_{a c} \phi(c, b) R_{c b} .
\end{aligned}
$$

In matrix form this recursion has the form (9), and it is easy to see that the solution is given by (10).

To use solution (8) for the case $H=D_{\alpha}$ or $H=N_{\alpha \beta}$, we must specify $\delta(n, T, f)$ and the matrix $M(n)$. Let the matrix with a 1 in the $(\alpha, \beta)$ th entry and 0 s elsewhere be denoted by $U(\alpha, \beta)$.

Corollary 1. In the case $H=D_{\alpha}$ we have $\phi=U(\alpha, \alpha), \operatorname{diag}(\psi)=U(\alpha, \alpha)$, and $\delta(n, T, f)=$ $T /(n+1)$. Solution (10) for $M(n)$ becomes

$$
M(n, a, b)=\sum_{\ell=0}^{n}\left(R^{n-\ell}\right)_{a \alpha} R_{\alpha b}^{\ell},
$$


and the mean value (8) is

$$
\mathrm{E}\left[D_{\alpha} 1(x(T)=b) \mid x(0)=a\right]=\sum_{n=0}^{\infty} \frac{T}{n+1}\left[\sum_{\ell=0}^{n}\left(R^{\ell}\right)_{a \alpha}\left(R^{n-\ell}\right)_{\alpha b}\right] \operatorname{Pois}(n ; \mu T) .
$$

Corollary 2. In the case $H=N_{\alpha \beta}$ we have $\phi=U(\alpha, \beta), \operatorname{diag}(\psi)=0$, and $\delta(n, T, f)=1$. Solution (10) for $M(n)$ becomes

$$
M(n, a, b)=\sum_{\ell=1}^{n}\left(R^{n-\ell}\right)_{a \alpha} R_{\alpha \beta} R_{\beta b}^{\ell-1}
$$

and the mean value (8) is

$$
\mathrm{E}\left[N_{\alpha \beta} 1(x(T)=b) \mid x(0)=a\right]=\sum_{n=1}^{\infty}\left[\sum_{\ell=1}^{n}\left(R^{n-\ell}\right)_{a \alpha} R_{\alpha \beta} R_{\beta b}^{\ell-1}\right] \operatorname{Pois}(n ; \mu T) .
$$

We note in passing that the mean value in (8) for the case $H=D_{\alpha}$ or $H=N_{\alpha \beta}$ can be obtained from the integral representations (3) and (4) on inserting the original uniformization result (5) for $P(t)$. As an example, we have

$$
\begin{aligned}
\mathrm{E}\left[D_{\alpha}\right. & 1(x(T)=b) \mid x(0)=a] \\
& =I(a, b, \alpha, \alpha) \\
& =\int_{0}^{T}\left[\sum_{i=0}^{\infty}\left(R^{i}\right)_{a \alpha} \frac{(\mu t)^{i}}{i !} \mathrm{e}^{-\mu t}\right]\left[\sum_{j=0}^{\infty}\left(R^{j}\right)_{\alpha b} \frac{(\mu(T-t))^{j}}{j !} \mathrm{e}^{-\mu(T-t)]} \mathrm{d} t\right. \\
& =\sum_{i=0}^{\infty} \sum_{j=0}^{\infty}\left(R^{i}\right)_{a \alpha}\left(R^{j}\right)_{\alpha b} \frac{T}{(i+j+1)} \mathrm{e}^{-\mu T} \frac{(\mu T)^{i+j}}{(i+j) !} \\
& =\sum_{n=0}^{\infty} \frac{T}{n+1}\left[\sum_{\ell=0}^{n}\left(R^{\ell}\right)_{a \alpha}\left(R^{n-\ell}\right)_{\alpha b}\right] \mathrm{e}^{-\mu T} \frac{(\mu T)^{n}}{n !} .
\end{aligned}
$$

This derivation, however, cannot be generalized to the most general form of $H$ in (6) as we do not have an integral representation in the general case.

For the case where $\phi\left(z_{1}, z_{2}\right)=\psi\left(z_{2}\right)$ and $f \equiv 1$, a recursion equivalent to (9) can be found in Narayana and Neuts (1992). The explicit forms (11) and (12) for $M(n)$ can be found in Bladt and Sørensen (2005).

\subsection{Computing covariances using uniformization}

In this subsection we consider two statistics $H$ and $\tilde{H}$ of the form (6), and we calculate $\mathrm{E}[H \tilde{H} 1(x(T)=b) \mid x(0)=a]$. We are particularly interested in the cases (2c)- (2e), where $H$ and $\tilde{H}$ are the times spent in a state and/or the numbers of jumps from one state to another. First, we introduce some more notation. Define

$$
\delta_{1}(n, T, f, \tilde{f})=\mathrm{E}[f(T X) \tilde{f}(T X)] \quad \text { and } \quad \delta_{2}(n, T, f, \tilde{f})=\mathrm{E}[f(T X) \tilde{f}(T Y)],
$$

where $(X, Y, 1-X-Y)$ follows a Dirichlet distribution with parameter $(1,1, n-1)$. We note that $\delta_{1}$ depends on $X$ only and that the marginal distribution of $X$ is a beta distribution with 
parameters $(1, n)$. Define the two matrices $M_{1}(n)$ and $L(n)$ as

$$
M_{1}(n, a, b)=\mathrm{E}\left[1\left(z_{n}=b\right)\left(\psi\left(z_{0}\right) \tilde{\psi}\left(z_{0}\right)+\sum_{i=1}^{n} \phi\left(z_{i-1}, z_{i}\right) \tilde{\phi}\left(z_{i-1}, z_{i}\right)\right) \mid z_{0}=a\right]
$$

and

$$
L(n, a, b)=\mathrm{E}\left[1\left(z_{n}=b\right) \sum_{i=1}^{n} \phi\left(z_{i-1}, z_{i}\right) \mid z_{0}=a\right]
$$

and define $\tilde{L}(n)$ similarly to $L(n)$ with $\phi$ replaced by $\tilde{\phi}$. Note that $M_{1}(n), L(n)$, and $\tilde{L}(n)$ have the same structure as $M(n)$ defined in (7), and, therefore, satisfy similar recursions and admit the same form of explicit solutions as in Theorem 1. Finally, define the matrix $M_{2}(n)$ as

$$
\begin{aligned}
M_{2}(n, a, b)=\mathrm{E}\left[1\left(z_{n}=b\right)(\right. & \psi\left(z_{0}\right) \sum_{i=1}^{n} \tilde{\phi}\left(z_{i-1}, z_{i}\right)+\tilde{\psi}\left(z_{0}\right) \sum_{i=1}^{n} \phi\left(z_{i-1}, z_{i}\right) \\
& \left.\left.+\sum_{\{i, j: i \neq j\}} \phi\left(z_{i-1}, z_{i}\right) \tilde{\phi}\left(z_{j-1}, z_{j}\right)\right) \mid z_{0}=a\right] .
\end{aligned}
$$

Theorem 2. (Products of general statistics.) Let $H$ and $\tilde{H}$ be two general statistics of the form (6). Then we have

$$
\begin{aligned}
& \mathrm{E}[H \tilde{H} 1(x(T)=b) \mid x(0)=a] \\
& \quad=\sum_{n=0}^{\infty}\left[\delta_{1}(n, T, f, \tilde{f}) M_{1}(n, a, b)+\delta_{2}(n, T, f, \tilde{f}) M_{2}(n, a, b)\right] \operatorname{Pois}(n ; \mu T) .
\end{aligned}
$$

The matrix $M_{1}(n)$ satisfies the recursion

$$
M_{1}(n)=M_{1}(n-1) R+R^{n-1}(\phi * \tilde{\phi} * R)
$$

with starting values $M_{1}(0)=\operatorname{diag}(\psi \tilde{\psi})$. The solution is

$$
M_{1}(n)=\operatorname{diag}(\psi \tilde{\psi}) R^{n}+\sum_{\ell=0}^{n-1} R^{\ell}(\phi * \tilde{\phi} * R) R^{n-1-\ell}
$$

The matrices $L(n)$ and $\tilde{L}(n)$ satisfy similar recursions and solutions with $\psi \tilde{\psi}$ replaced by 0 and $\phi \tilde{\phi}$ replaced by $\phi$ or $\tilde{\phi}$. The matrix $M_{2}(n)$ satisfies the recursion

$$
\begin{aligned}
M_{2}(n)= & M_{2}(n-1) R+\operatorname{diag}(\psi) R^{n-1}(\tilde{\phi} * R)+\operatorname{diag}(\tilde{\psi}) R^{n-1}(\phi * R) \\
& +\tilde{L}(n-1)(\phi * R)+L(n-1)(\tilde{\phi} * R) .
\end{aligned}
$$

The solution to the latter recursion can be written as

$$
\begin{aligned}
M_{2}(n)= & \operatorname{diag}(\psi) \sum_{\ell=0}^{n-1} R^{\ell}(\tilde{\phi} * R) R^{n-1-\ell}+\operatorname{diag}(\tilde{\psi}) \sum_{\ell=0}^{n-1} R^{\ell}(\phi * R) R^{n-1-\ell} \\
& +\sum_{\ell=0}^{n-2} \sum_{s=0}^{n-2-\ell} R^{\ell}\left[(\tilde{\phi} * R) R^{s}(\phi * R)+(\phi * R) R^{s}(\tilde{\phi} * R)\right] R^{n-2-\ell-s} .
\end{aligned}
$$


Proof. Conditional on the number of jumps $n$, we can divide the product of $H$ and $\tilde{H}$ according to terms where the interarrival times are the same or different:

$$
\begin{aligned}
H \tilde{H}= & \psi\left(z_{0}\right) \tilde{\psi}\left(z_{0}\right) f\left(W_{1}\right) \tilde{f}\left(W_{1}\right)+\sum_{i=1}^{n} \psi\left(z_{0}\right) \tilde{\phi}\left(z_{i-1}, z_{i}\right) f\left(W_{1}\right) \tilde{f}\left(W_{i+1}\right) \\
& +\sum_{i=1}^{n} \tilde{\psi}\left(z_{0}\right) \phi\left(z_{i-1}, z_{i}\right) \tilde{f}\left(W_{1}\right) f\left(W_{i+1}\right) \\
& +\sum_{i=1}^{n} \phi\left(z_{i-1}, z_{i}\right) \tilde{\phi}\left(z_{i-1}, z_{i}\right) f\left(W_{i+1}\right) \tilde{f}\left(W_{i+1}\right) \\
& +\sum_{\{i, j: i \neq j\}} \phi\left(z_{i-1}, z_{i}\right) \tilde{\phi}\left(z_{j-1}, z_{j}\right) f\left(W_{i+1}\right) \tilde{f}\left(W_{j+1}\right) .
\end{aligned}
$$

Taking means with respect to $\left(W_{1}, \ldots, W_{n+1}\right)$ conditioned on $W_{1}+\cdots+W_{n+1}=T$ and conditioning on $z_{0}=a$, we obtain

$$
\begin{aligned}
& \mathrm{E}[H \tilde{H} 1(x(T)=b) \mid x(0)=a, J=n] \\
& \quad=\delta_{1}(n, T, f, \tilde{f}) M_{1}(n, a, b)+\delta_{2}(n, T, f, \tilde{f}) M_{2}(n, a, b) .
\end{aligned}
$$

The derivation of the recursion for $M_{1}(n)$ is as in the proof of Theorem 1 for $M(n)$ with $\psi$ replaced by $\psi \tilde{\psi}$ and with $\phi$ replaced by $\phi * \tilde{\phi}$.

To establish the recursion for $M_{2}(n)$, we proceed as in the proof of Theorem 1, dividing the mean value according to the value of $z_{n-1}$. This gives, after some manipulations,

$$
\begin{aligned}
M_{2}(n, a, b)=\sum_{c=1}^{m} & {\left[M_{2}(n-1, a, c) R_{c b}+\left(R^{n-1}\right)_{a c}[\psi(a) \tilde{\phi}(c, b)+\tilde{\psi}(a) \phi(c, b)] R_{c b}\right.} \\
& \left.+\tilde{L}(n-1, a, c) \phi(c, b) R_{c b}+L(n-1, a, c) \tilde{\phi}(c, b) R_{c b}\right] .
\end{aligned}
$$

The starting value of the recursion is $M_{2}(0)=0$. The recursions for $L(n)$ and $\tilde{L}(n)$ are derived as for $M(n)$ in the proof of Theorem 1 . The solutions of the latter recursions correspond to the solution of the recursion for $M(n)$. Using these, it can be shown that the solution of the recursion for $M_{2}(n)$ is as stated in the theorem.

From the properties of the beta and Dirichlet distributions we obtain the following corollary.

Corollary 3. In the case where both $H$ and $\tilde{H}$ correspond to either the times spent in a state or the numbers of jumps between two states the covariance term can be found from Theorem 2 on using

$$
\delta_{1}(n, T, f, \tilde{f})= \begin{cases}1 & \text { if } f=\tilde{f} \equiv 1, \\ \frac{T}{(n+1)} & \text { if } f(t)=t \text { and } \tilde{f} \equiv 1 \text { or } f \equiv 1 \text { and } \tilde{f}(t)=t, \\ \frac{2 T^{2}}{(n+1)(n+2)} & \text { if } f(t)=\tilde{f}(t)=t,\end{cases}
$$


and

$$
\delta_{2}(n, T, f, \tilde{f})= \begin{cases}1 & \text { if } f=\tilde{f} \equiv 1, \\ \frac{T}{(n+1)} & \text { if } f(t)=t \text { and } \tilde{f} \equiv 1 \text { or } f \equiv 1 \text { and } \tilde{f}(t)=t, \\ \frac{T^{2}}{(n+1)(n+2)} & \text { if } f(t)=\tilde{f}(t)=t .\end{cases}
$$

For the case where $\phi\left(z_{1}, z_{2}\right)=\psi\left(z_{2}\right), f \equiv 1, \tilde{\phi}=\phi$, and $\tilde{f}=f$, a recursion equivalent to the recursion for $M_{2}(n)$ can be found in Narayana and Neuts (1992). Generally, the formulae of this section appear to be new. Bladt and Sørensen (2009) used numerical differentiation (with respect to the entries of the rate matrix $Q$ ) to find the covariance terms of this section.

\subsection{Computing distributions using uniformization}

In this subsection we use uniformization to derive the distribution of the number of state changes and the time spent in states using uniformization.

Siepel et al. (2006) used uniformization to derive the distribution of the total number of substitutions $N=\sum_{i=1}^{J} 1\left(z_{i-1} \neq z_{i}\right)$. Other statistics of interest are the number of substitutions $N_{\alpha \beta}=\sum_{i=1}^{J} 1\left(\left(z_{i-1}, z_{i}\right)=(\alpha, \beta)\right)$ between two different states $\alpha$ and $\beta$, and the number of visits $N_{\alpha}=\sum_{i=1}^{J} 1\left(z_{i-1}=\alpha\right)$ to a state $\alpha$. In this section we consider a general count statistic

$$
N_{H}=\psi\left(z_{0}\right)+\sum_{i=1}^{J} \phi\left(z_{i-1}, z_{i}\right)
$$

where entries in both $\phi$ and $\psi$ can be 0 or 1 only. We let $\mathrm{P}(k, n)$ be the $m \times m$ matrix with entries

$$
\mathrm{P}(k, n, a, b)=\mathrm{P}\left(\psi\left(z_{0}\right)+\sum_{i=1}^{n} \phi\left(z_{i-1}, z_{i}\right)=k, z_{n}=b \mid z_{0}=a\right) .
$$

Thus, $\mathrm{P}(k, n, a, b)$ is the probability of recording $k$ counts of interest when the Markov chain starts in state $a$ and must be in state $b$ at time $n$. Note that

$$
\mathrm{P}(0,0)=\operatorname{diag}(1-\psi) \quad \text { and } \quad \mathrm{P}(1,0)=\operatorname{diag}(\psi),
$$

and that $\mathrm{P}(k, n)=0$ for $k>n+1$.

Theorem 3. Let $N_{H}$ be given as in (13) where entries in both $\phi$ and $\psi$ can be 0 or 1 only. We have

$$
\mathrm{P}\left(N_{H}=k, x(T)=b \mid x(0)=a\right)=\sum_{n=0}^{\infty} \mathrm{P}(k, n, a, b) \operatorname{Pois}(n ; \mu t),
$$

where, for $n \geq 1$ and $1 \leq k \leq n+1$, the matrix $\mathrm{P}(k, n)$ is given by the recursion

$$
\mathrm{P}(k, n)=\mathrm{P}(k-1, n-1)(R * \phi)+\mathrm{P}(k, n-1)(R *(1-\phi)) .
$$

Proof. The uniformization method gives

$$
\mathrm{P}\left(N_{H}=k, x(T)=b \mid x(0)=a\right)=\sum_{n=0}^{\infty} \mathrm{P}(k, n, a, b) \operatorname{Pois}(n ; \mu t),
$$

where $\mathrm{P}(k, n)$ is defined in (14). 
The recursion for $\mathrm{P}(k, n, a, b)$ is derived by dividing according to the value of $z_{n-1}$, i.e.

$$
\begin{aligned}
\mathrm{P}(k, n, a, b) & \\
= & \sum_{c=1}^{m} \mathrm{P}\left(\psi\left(z_{0}\right)+\sum_{i=1}^{n} \phi\left(z_{i-1}, z_{i}\right)=k, z_{n-1}=c, z_{n}=b \mid z_{0}=a\right) \\
= & \sum_{c=1}^{m} \mathrm{P}\left(\psi\left(z_{0}\right)+\sum_{i=1}^{n-1} \phi\left(z_{i-1}, z_{i}\right)=k-1, z_{n-1}=c \mid z_{0}=a\right) \phi(c, b) R_{c b} \\
& +\sum_{c=1}^{m} \mathrm{P}\left(\psi\left(z_{0}\right)+\sum_{i=1}^{n-1} \phi\left(z_{i-1}, z_{i}\right)=k, z_{n-1}=c \mid z_{0}=a\right)(1-\phi(c, b)) R_{c b} \\
= & \sum_{c=1}^{m} \mathrm{P}(k-1, n-1, a, c) \phi(c, b) R_{c b}+\sum_{c=1}^{m} \mathrm{P}(k, n-1, a, c)(1-\phi(c, b)) R_{c b},
\end{aligned}
$$

on using the fact that $\phi(c, b)$ is either 1 or 0 .

Let us illustrate the result of the theorem for the case considered in Siepel et al. (2006). Thus, we consider the total number of substitutions, $N=\sum_{i=1}^{J} 1\left(z_{i-1} \neq z_{i}\right)$. For this case, $\mathrm{P}(0,0)=\operatorname{diag}(1,1, \ldots, 1), \mathrm{P}(1,0)=0$, and the recursion is given by

$$
\mathrm{P}(k, n, a, b)=\sum_{\{c: c \neq b\}} \mathrm{P}(k-1, n-1, a, c) R_{c b}+\mathrm{P}(k, n-1, a, b) R_{b b} .
$$

The interpretation of the recursion is quite clear. We divide the probability according to the last jump being from a state $c$ to the state $b$, and the two terms in the recursion correspond to the last jump being a real substitution $(c \neq b)$ or a virtual substitution $(c=b)$.

In the case of the number of transitions from state $\alpha$ to state $\beta$ given by the statistic $N_{\alpha \beta}$ we obtain the starting values $\mathrm{P}(k, 0, a, b)=0$ for $k \geq 0$. For $n \geq 1$ and $1 \leq k \leq n+1$, we have the recursion

$$
\mathrm{P}(k, n, a, b)= \begin{cases}\mathrm{P}(k-1, n-1, a, \alpha) R_{\alpha b}+\sum_{\{c: c \neq \alpha\}} \mathrm{P}(k, n-1, a, c) R_{c b} & \text { if } b=\beta, \\ \sum_{c=1}^{m} \mathrm{P}(k, n-1, a, c) R_{c b} & \text { if } b \neq \beta .\end{cases}
$$

Again, the interpretation is quite clear. The probability is calculated according to the last jump being from a state $c$. If the ending state $b$ is different from $\beta$ then the jump is never from $\alpha$ to $\beta$; this is the last case. If the ending state is $b=\beta$ then the jump is from $\alpha$ to $\beta$ when $c=\beta$. These considerations justify the first case.

As a final application of the uniformization method, we consider a sum of interarrival times like, for example, the time spent in a state $\alpha, D_{\alpha}=\sum_{i=0}^{J} 1\left(z_{i}=\alpha\right)\left(T_{i+1}-T_{i}\right)$. Such a statistic has a mixed distribution with point probabilities at 0 and $T$, and has a continuous distribution between these two points. The trick to handle this statistic is that, conditional on $J=n$, the distribution depends on the number of terms in the sum only. Furthermore, the distribution of the number of terms is given through $\mathrm{P}(k, n)$ from Theorem 3 . In the theorem below we treat a general statistic of the form

$$
D_{H}=\psi\left(z_{0}\right) T_{1}+\sum_{i=1}^{J} \phi\left(z_{i-1}, z_{i}\right)\left(T_{i+1}-T_{i}\right),
$$


where both $\phi$ and $\psi$ can be 0 and 1 only. We let $f(t ; a, b)$ be the conditional density of $D_{H}$ given $x(0)=a$ on the set $x(T)=b$ :

$$
\mathrm{P}\left(0<D_{H}<t, x(T)=b \mid x(0)=a\right)=\int_{0}^{t} f(y ; a, b) \mathrm{d} y, \quad t<T .
$$

In the theorem below we let $f_{\mathrm{B}}\left(u ; \lambda_{1}, \lambda_{2}\right)$ be the beta density given by

$$
f_{\mathrm{B}}\left(u ; \lambda_{1}, \lambda_{2}\right)=\frac{\Gamma\left(\lambda_{1}+\lambda_{2}\right)}{\Gamma\left(\lambda_{1}\right) \Gamma\left(\lambda_{2}\right)} u^{\lambda_{1}-1}(1-u)^{\lambda_{2}-1} .
$$

Theorem 4. The distribution of $D_{H}$ in (15) is given by

$$
\begin{gathered}
\mathrm{P}\left(D_{H}=0, x(T)=b \mid x(0)=a\right)=\sum_{n=0}^{\infty} \mathrm{P}(0, n, a, b) \operatorname{Pois}(n ; \mu T), \\
\mathrm{P}\left(D_{H}=T, x(T)=b \mid x(0)=a\right)=\sum_{n=0}^{\infty} \mathrm{P}(n+1, n, a, b) \operatorname{Pois}(n ; \mu T), \\
f(t ; a, b)=\sum_{n=1}^{\infty} \sum_{k=1}^{n} \frac{1}{T} f_{\mathrm{B}}\left(\frac{t}{T} ; k, n-k+1\right) \mathrm{P}(k, n, a, b) \operatorname{Pois}(n ; \mu T),
\end{gathered}
$$

where $\mathrm{P}(k, n, a, b)$ is given in Theorem 3.

Proof. Let $N_{H}=\psi\left(z_{0}\right)+\sum_{i=1}^{J} \phi\left(z_{i-1}, z_{i}\right)$. Using the uniformization method, we condition on $J=n$. For $D_{H}$ to be 0 , we must have $N_{H}=0$, and the probability of this is given by $\mathrm{P}(0, n, a, b)$. Similarly, for $D_{H}$ to be $T$, we must have $N_{H}=n+1$, and the probability of this is given by $\mathrm{P}(n+1, n, a, b)$. For $0<N_{H}=k<(n+1)$, the density of $D_{H}$ is the density of $\sum_{j=1}^{k} W_{i}$ given that $\sum_{j=1}^{n+1} W_{i}=T$. In this conditional distribution $(1 / T) \sum_{j=1}^{k} W_{i}$ has a beta distribution with parameters $(k, n-k+1)$. This then gives the formula for $f(t ; a, b)$ on using the fact that the conditional probability of $N_{H}=k$ is $\mathrm{P}(k, n, a, b)$.

\section{Integrals of matrix exponentials}

For the time spent in a state $D_{\alpha}$ or the number of jumps between two states $N_{\alpha \beta}$, the first two moments can be calculated from the integral representations (3) and (4). These representations and an eigenvalue decomposition of the rate matrix were used in Section 2. When an eigenvalue decomposition is not available we derived in Section 3 alternative expressions based on the uniformization method. There is, however, a third approach to the calculation of integrals of matrix exponentials as those in (3) and (4). The purpose of this section is to draw attention to the theory developed in Van Loan (1978) for calculating such integrals. We describe the method of Van Loan (1978) in its most simple form.

Consider the problem of evaluating the integral

$$
\int_{0}^{T} \mathrm{e}^{Q(T-t)} B \mathrm{e}^{Q t} \mathrm{~d} t
$$

where $B$ is a matrix of the same dimension as $Q$. The special case with $B=U(\alpha, \beta)$ gives the integral in (3). For evaluating this integral, Van Loan (1978) introduced a matrix $A$, with a dimension twice that of $Q$ :

$$
A=\left[\begin{array}{ll}
Q & B \\
0 & Q
\end{array}\right]
$$


The structure of $A$ implies that the matrix exponential must be of the form

$$
\mathrm{e}^{A t}=\left[\begin{array}{cc}
F(t) & G(t) \\
0 & F(t)
\end{array}\right] \text { with } \quad F(0)=I \quad \text { and } \quad G(0)=0 .
$$

Using the fact that $\mathrm{de}^{A t} / \mathrm{d} t=A \mathrm{e}^{A t}$, we obtain the equation

$$
\left[\begin{array}{cc}
F^{\prime}(t) & G^{\prime}(t) \\
0 & F^{\prime}(t)
\end{array}\right]=\left[\begin{array}{cc}
Q & B \\
0 & Q
\end{array}\right]\left[\begin{array}{cc}
F(t) & G(t) \\
0 & F(t)
\end{array}\right] .
$$

We thus have two differential equations to solve. A homogeneous linear differential equation

$$
F^{\prime}(t)=Q F(t) \quad \text { with } \quad F(0)=I
$$

(the solution is $F(t)=\mathrm{e}^{Q t}$ ), and an inhomogeneous linear differential equation

$$
G^{\prime}(t)=Q G(t)+B F(t) \quad \text { with } \quad G(0)=0 .
$$

The inhomogeneous linear differential equation has the solution

$$
G(t)=\int_{0}^{t} \mathrm{e}^{(t-x) Q} B F(x) \mathrm{d} x=\int_{0}^{t} \mathrm{e}^{(t-x) Q} B \mathrm{e}^{Q x} \mathrm{~d} x .
$$

Thus, integral (16) is the upper-right corner in $\mathrm{e}^{A t}$.

A number of approaches exist for evaluating $\mathrm{e}^{A t}$ and are implemented in software packages; see Moler and Van Loan (2003) for a review. Calculating the matrix exponential $\mathrm{e}^{A t}$ therefore provides a very direct way of calculating the desired integral representation. The only caveat is the accuracy of the matrix exponential utility provided by the software.

To handle integral (4), we can use the part of Van Loan's methodology concerning integrals of the type

$$
\int_{0}^{T} \int_{0}^{t} \mathrm{e}^{Q(T-u)} B_{1} \mathrm{e}^{Q(t-u)} B_{2} \mathrm{e}^{Q u} \mathrm{~d} u \mathrm{~d} t .
$$

This integral is given by the upper-right corner of $\mathrm{e}^{A t}$ with

$$
\left[\begin{array}{ccc}
Q & B_{1} & 0 \\
0 & Q & B_{2} \\
0 & 0 & Q
\end{array}\right]
$$

The reader is encouraged to consult Van Loan (1978) for the proof.

\section{Discussion}

We have presented three approaches for calculating expectations of summary statistics for endpoint-conditioned continuous-time Markov chains. The eigenvalue decomposition method and Van Loan's method both take the integral representations (3) and (4) as a starting point. The integral representations (3) and (4) cover the most important summary statistics (time spent in a state and number of jumps from one state to another). However, in general, a summary statistic $H$ of the form (6) does not necessarily admit an integral representation. In Section 3 we provided a framework for calculating properties of general summary statistics. The framework is based 
on the uniformization method, and, as shown in Theorem 1, the calculation is decomposed into three simple parts that are each easy to handle.

All three methods of calculating properties of summary statistics are in principle easy to implement, but they all have their limitations. The method based on an eigenvalue decomposition becomes rather involved if the eigenvalues are not real. (However, we would like to emphasize that a reversible substitution process always admits real eigenvalues.) Van Loan's method seems very attractive but requires access to a reliable matrix exponentiation software package. Uniformization is the most general method, but requires that an infinite sum is truncated. A discussion of truncation error can be found in Narayana and Neuts (1992) and Grassmann (1993).

\section{References}

Ball, F. AND Milne, R. K. (2005). Simple derivations of properties of counting processes associated with Markov renewal processes. J. Appl. Prob. 42, 1031-1043.

Bladt, M. and Sørensen, M. (2005). Statistical inference for discretely observed Markov jump processes. $J$. $R$. Statist. Soc B 67, 395-410.

Bladt, M. AND SøREnSEn, M. (2009). Efficient estimation of transition rates between credit ratings from observations at discrete time points. Quant. Finance 9, 147-160.

Bladt, M., Meini, B., Neuts, M. F. and Sericola, B. (2002). Distributions of reward functions on continuous-time Markov chains. In Matrix-Analytic Methods, ed. G. Latouche, World Scientific, River Edge, NJ, pp. $39-62$.

Dempster, A. P., Laird, N. M. ANd Rubin, D. B. (1977). Maximum likelihood from incomplete data via the EM algorithm. J. R. Statist. Soc B 39, 1-38.

Grassmann, W. K. (1993). Rounding errors in certain algorithms involving Markov chains. ACM Trans. Math. Software 19, 496-508.

GutTorp, P. (1995). Stochastic Modeling of Scientific Data. Chapman and Hall, London.

Hobolth, A. And Jensen, J. L. (2005). Statistical inference in evolutionary models of DNA sequences via the EM algorithm. Statist. Appl. Genet. Molec. Biol. 4, 20pp.

Holmes, I. AND Rubin, G. M. (2002). An expectation maximization algorithm for training hidden substitution models. J. Molec. Biol. 317, 753-764.

Jensen, A. (1953). Markoff chains as an aid in the study of Markoff processes. Skand. Aktuarietidskr. 36, 87-91.

Klosterman, P. S. et al. (2006). XRate: a fast prototyping, training and annotation tool for phylo-grammars. BMC Bioinformatics 7, 25pp.

Kosiol, C., Holmes, I. ANd Goldman, N. (2007). An empirical codon model for protein sequence evolution. Molec. Biol. Evol. 24, 1464-1479.

Metzner, P., Horenko, I. And Schütte, C. (2007). Generator estimation of Markov jump processes based on incomplete observations nonequidistant in time. Phys. Rev. E 76, 066702, 8pp.

Minin, V. N. AND SuCHARD, M. A. (2008). Counting labeled transitions in continuous-time Markov models of evolution. J. Math. Biol. 56, 391-412.

Moler, C. AND VAN LOAN, C. (2003). Nineteen dubious ways to compute the exponential of a matrix, twenty-five years later. SIAM Rev. 45, 3-49.

NaRAYANA, S. AND Neuts, M. F. (1992). The first two moment matrices of the counts for the Markovian arrival process. Commun. Statist. Stoch. Models 8, 459-477.

Ross, S. M. (1983). Stochastic Processes. John Wiley, New York.

Siepel, A., Pollard, K. S. And Haussler, D. (2006). New methods for detecting lineage-specific selection. In Research in Computational Molecular Biology (Lecture Notes Bioinformatics 3909), eds A. Apostolico et al., Springer, Berlin, pp. 190-205.

VAN LOAN, C. F. (1978). Computing integrals involving the matrix exponential. IEEE Trans. Automatic Control 23, 395-404. 